Proc. Indian Acad. Sci. (Chem. Sci.), Vol. 106, No. 5, October 1994, pp. 1253-1258.

(C) Printed in India.

\title{
Control of lipid microstructures by molecular design and its implications
}

\author{
SANTANU BHATTACHARYA \\ Department of Organic Chemistry, Indian Institute of Science, Bangalore 560012, India
}

\begin{abstract}
An overview of the current trends in the lipid design for specific applications has been presented. Lipids with different surface charge and hydrophobic backbone undergo aggregation to produce lamellae or bilayer and multilayer vesicles in aqueous media. Various aspects of present development of chiral superstructures and enzyme-mimics have been discussed. Utility of these molecules for potential applications in immunomodulation and sustained drug-delivery systems is also summarized.
\end{abstract}

Keywords. Lipids; vesicles; liposomes; chiral superstructures; immunomodulators; sustainedrelease systems.

\section{Introduction}

Bioorganic approaches to the design and construction of synthetic cells have been the focus of much current research (Fendler 1982; Murakami and Kikuchi 1991). The barrier that defines the interior and exterior of a living cell is a cell membrane. Presumably, the primitive forms of life originated from organized assembly of amphiphilic molecules such as lipids which ultimately gave rise to plasma membranes (Fendler 1984; Ringsdorf et al 1988). Biological cell membranes are complex entities. Although lipids are predominant constituents of biological membranes, different biologically active macromolecules such as proteins, carbohydrates, steroids etc. also share the same habitat i.e., the cell membrane. Their important functions generally derive from their propensity to self-organize. Such self-assembly provides internal mesophases (smectic) with controlled sizes, shapes, fluidities, and micro-environments. In this capacity, various bioactive macromolecules carry out important biological functions such as transport, transduction of signals, separation of materials across membrane bilayers, fusion, exo- and endocytosis etc. within the protective matrix of cell membranes (Cevc and Marsh 1987; Slater and Huang 1992).

\section{Molecular aggregate of lipids in aqueous media}

Lipids are comparatively unique biomolecules in the sense that they are generally not polymeric in nature. Instead, several molecules of lipids cooperatively organize through non-covalent interactions. Lipids and related molecules usually have both a watersoluble and fat-soluble domain and hence they are amphiphilic in nature (Fendler 1980; Shobha and Balasubramanian 1986; Shobha et al 1989; Sundar et al 1991). The self-organization takes place in water because of the fact that they are amphiphilic 
in nature. Various kinds of microscopic structures are produced from the self-assembly of lipid molecules. As a consequence of hydrophobic forces in the lipophilic region and electrostatic and hydrogen bonding interactions around aqueous interfaces, these molecules supramolecularly aggregate to produce closed bags or spheres made up of one or more lipid bilayers. These are known as liposomes or vesicles (Brockerhoff 1977). Vesicles composed of synthetic lipids of defined chemical structures are the simplest functional models of membranes (Chapman 1975; Raison 1980). Vesicles contain entrapped water within the enclosures of bilayer mesophases. Generally, vesicles could be unilamellar (one bilayer leaflet) or multilamellar (several bilayers) in organization with apparent spherical or ellipsoidal shapes. Vesicles have been shown to provide effective barrier for transport of water-soluble solutes or ions. However, passage of hydrophilic substances across the bilayer membranes would depend on lipid molecular structure, kind of microstructure produced as a consequence of selfassembly of these lipids and the temperature at which the transport is measured. This is because generally vesicles display a gel to liquid crystalline thermotropic phase transition (Huang and Mason 1986; Small 1986). For a lipid of given chemical structure, there has to be a characteristic critical temperature for such transition which is generally known as melting temperature, Tm (McElhaney 1986; Ślvius 1991; Menger et al 1993). The basic structural and physical features of synthetic amphiphiles are not only important determinants for the development of new supramolecular assemblies and networks (Fuhrhop 1993) but are also crucial for sustaining biological macromolecules such as membrane proteins in a native folded conformation (Knox et al, in preparation). In order to understand complex lipid-protein interactions, it is therefore necessary to replace complex composition of the cell membranes with known quantities of structurally defined amphiphilic detergents or lipid (Schleicher et al 1987). Thus, reconstitution of rhodopsin without denaturation could be successfully carried out in partially polymerized vesicular suspensions (Tyminski et al 1988).

\section{Molecular design of novel lipids}

The natural membrane forming lipids are either anionic, neutral, or zwitterionic in nature and generally contain two fatty acyl chains. More than a decade ago, Kunitake and co-workers demonstrated that upon sonic dispersal in aqueous buffer, dialkyl dimethylammonium ions could form closed structures that are remarkably similar to those of naturally occurring phospholipids (Kunitake and Okahata 1977). Due to structural simplicity and easier amenability to synthetic manipulations, large number of reports have appeared in subsequent literature concerning structure-property relationship in other kinds of new lipids (Murakami et al 1980; Sudholter et al 1980; Fuhrhop and Matheiu 1984; Rupert et al 1986; Kaler et al 1989; Fukuda et al 1990; Zana and Talmon 1993). Now, several preparations of vesicles from fully cationic, anionic or zwitterionic lipids of completely synthetic, non-biological origin have been characterized. It has been realized that bilayer aggregates can also be generated from amphiphiles that have rigid azobenzene, biphenyl or diphenylazomethine moieties inserted into a single hydrophobic tail (Kunitake et al 1981, 1984; Cho and Park 1987; Kunitake 1992). 


\section{Chiral membranous assemblies, helical and tubular microstructures}

Kunitake and co-workers have successfully employed chiral amino acids as useful modules in the design of bilayer forming molecules (Nakashima et al 1989; Ishikawa et al 1990). Such synthetic chiral bilayers composed of single hydrocarbon or double hydrocarbon chain amphiphiles display pronounced circular dichroism as a consequence of the formation of higher order supramolecular organizations. In this case, the amphiphiles were first dispersed in vesicular form by ultrasonication and then left standing for several hours. The vesicular structures budded out to form short rodlike structures which eventually got converted into twisted helical ribbons.

Yanagawa and co-workers have developed double chain nucleotide derivatives (Yanagawa et al 1989). Upon sonication, dimyristoyl 5'-phosphatidyl-cytidine formed vesicles, which got slowly transformed into circular helical strands with hydrodynamic radii of $25-75 \mathrm{~nm}$ in presence of $0.2 \mathrm{M} \mathrm{KCl}$. Interestingly, at lower $\mathrm{KCl}$ concentrations $(0.01-0.05 \mathrm{M})$, linear helical strands were seen. On the other hand, at higher $\mathrm{KCl}$ concentrations $(0.5-1.0 \mathrm{M})$, the vesicles maintained their morphological integrity.

Ultrathin molecular bilayer ribbons could be generated from solutions of $n$-octyl-Dgalactonamide (Fuhrhop et al 1988, 1990). In contrast, the corresponding dispersions of diastereomeric amphiphile, $n$-octyl-D-gluconamide produced apparent rodlike structures. The former is about two orders of magnitude less soluble than the latter and consequently a mixture of both straight and twisted ribbons are produced. Interestingly, solubilization of $n$-octyl-D-gluconomide in ortho xylene in which it is much less soluble than in water results in helical ribbons (Fuhrhop et al 1990). Long thin tubules could be obtained from amphiphiles, which contain diacetylenic units in their long hydrocarbon chains (Singh et al 1988; Merkowitz and Singh 1991). Tubules can be produced either by gradual cooling of resulting vesicular dispersions or by mixing water to ethanolic solution of such lipids. It has also been realized that the specific morphology of the tubules is a critical function of the lipid headgroup chosen. Thus, substitution of a choline headgroup with a smaller hydroxyethanol headgroup results in the formation of tight tubules with a pronounced helical structure (Gaber 1990).

\section{Functional molecular assemblies as enzyme analogues}

Studies on the stereoselective hydrolysis of peptide esters in various lipid/surfactant coaggregates, allow one to reveal that considerable stereochemical control is indeed attainable by regulation of the composition of coaggregates, temperature and ionic strength (Lehn 1988; Ueoka et al 1988). Appropriate "fine-tuning" of the aggregate microenvironments can produce significant enantioselectivity in such reactions. Such findings have been attributed to the optimization of the enzyme model conformation in the coaggregate ensembles (Ueoka et al 1992).

Hamachi et al (1991) have shown that a water-soluble heme protein, myoglobin could be embedded within the interbilayer spaces of synthetic amphiphilic assemblies without denaturation. The orientation of protein molecules in such reconstituted systems is believed to be maintained as a consequence of favourable electrostatic interactions between the positively charged side chain residues from the polypeptide chains and the negatively charged phosphate functions of the amphiphiles on the 
membrane surface (Kunitake et al 1990; Hamachi et al 1991). Interestingly, the myoglobin molecules in such a matrix retain their redox capacity also.

\section{Glycolipid analogues as potential immunomodulators}

Due to their residence at the cell surfaces, glycolipids can act as ideal systems for binding antibodies, bacteria, lectins, toxins or viruses (Paulson 1985). As a consequence, they play a major role in cell-to-cell communication and interactions with cell adhesion molecules (CAM) (Edleman 1988). Several hundreds of glycolipids are known from natural origin. If provision of a cellular matrix for biological events is the only purpose for their evolution, there is little to justify why nature has spent so much metabolic energy to produce so many of them (Boggs 1986). This points to more important roles which may not be apparent at this point of time. Based on their general structural features, they can be classified into two groups, the glycoglycerolipids and glycosphingolipids.

Lockhoff and co-workers developed a series of structurally defined, simple glycolipid analogs in view of their fundamental importance as cell-wall amphiphiles by joining lipophilic hydrocarbon chains with carbohydrate headgroups (Paulson et al 1980). While natural glycolipids produce in general an immunosuppressive effect, these novel glycolipid analogs elicit significant immunostimulation (Lockhoff 1991). As a result these systems are excellent candidates for treating patients suffering from immunoprophylaxis (Lockhoff 1991).

Although, the molecular mechanism that are responsible for such immunological activities of glycolipid analogs are still unknown, there is evidence that even subtle structural changes in the polar headgroups of glycolipids and phospholipids have strong effects on mutual miscibility in solid and liquid crystalline mesophases. Since these factors also control the membrane fluidity, the conformation of receptor proteins in such membrane matrix can also get altered (Koynova et al 1988).

\section{Reactivity control and regulated delivery systems}

The molecular structures of the amphiphiles and lipids and the physical states of their microstructures can determine various properties. Thus, liposomes of one molecular structure can effectively retain hydrophilic solutes in a way better than the liposomes produced from another lipid. Such abilities to control and regulate the reactivities of two species or sustaining a material inside its aqueous millieu have important implications (Surolia and Bachhawat 1977; Curatolo et al 1985; Smith et al 1994). In particular, this aspect is crucial for utilization of vesicles as entities for encapsulation of bioactive substances and drugs and to obtain stabilized drug carriers. Uptake of liposomes by the reticuloendothelial system (RES), mainly the fixed macrophages of liver and spleen, actually proved to be beneficial for those anxious to deliver drugs to macrophages. For instance, this is useful in the treatment of certain microbial diseases, enzyme deficiencies or metal-storage diseases, the activation of tumoricidal macrophages and immuno-potentiation of vaccines (Gregoriadis 1988; Bachhawat 1991). As expected the composition and other properties of liposomes (vesicle size and charge, and the extent of hydration of the headgroup, for example) are likely to play important roles in their interactions with and possible penetration into epidermis. Agents incorporated in the vesicle bilayer presumably reduce the energy barrier of their interaction with its epidermal lipidic environment and thus 
promote coalescence. Evidence obtained so far (Ghyczy and Niemann 1992) indicates that appropriately tailored liposomes usually. with diameters of $200 \mathrm{~nm}$ or less do indeed enter the epidermis. These are to be seen as deformed spherical structures between the corneocytes of the stratum corneum (Junginger et al 1991). The list of vesicle entrapped molecules which are claimed to assist the epidermis to function (especially for ageing women) is long. These include peptides, carbohydrates, proteins and special lipids, among others. Some of these are believed to mitigate harmful effects of free radicals and also inhibit their generation as a result of the combined action of oxygen and UV radiation etc. (Gregoriadis 1994). Many new cosmetic industries are now interested in developing receptor-mediated targeted drug delivery. These new liposomes are equipped with a homing device (rhamnose) specific for keratinocytes expressing the rhamnose receptor. The idea is that such liposomes would interact with the target cell more effectively and deliver their active agent more efficiently. It however, appears that exploitation of liposomal vesicles, is only in its infancy. Consequently, there is a lot of scope for newer opportunities (Gregoriadis 1994).

\section{Conclusions}

Clearly, there are two distinct facets which are becoming conspicuous in such research initiatives. One involves fundamental approaches involving bioorganic and biological investigations of defined lipid assemblies and their interactions with proteins and other lipid interactable macromolecules. The second approach attempts to exploit new systems for developing novel materials for nanotechnology, biotechnological purposes, pharmaceutical formulations and drug delivery.

The purpose of this article is to review current trends in the molecular design of lipids and their potential from a bioorganic point of view using literature descriptions and explanations that strive for brevity, clarity and simplicity rather than for completeness and rigour. Obviously, it is beyond the scope of this article to encompass many other important aspects. For a specific research subject, one needs to go to the primary literature for substantive details. Nevertheless, it appears clear from foregoing discussions that the stage is ready for exciting new findings. Near future will witness multifarious applications involving designed lipids and amphiphiles both in biotechnology and in materials science, two most emerging disciplines of the present time (Schnur 1993).

\section{References}

Bachhawat B K 1991 Liposome technology. In The CSIR Distinguished Lecture Series (New Delhi: CSIR)

Boggs J M 1986 Biochem. Cell Biol. 6450

Brockerhoff H 1977 Molecular design of membrane lipids. In Bioorganic chemistry (ed.) E E Van Tamelen (New York: Academic Press) vol. 3, chap. 1

Cevc G and Marsh D 1987 Phospholipid bilayers (New York: Wiley-Interscience)

Chapman D 1975 Biomembranes 731

Cho I and Park J G 1987 Chem. Lett. 977

Curatolo W, Bali A and Gupta C M 1985 J. Pharm. Sci. 741255

Edleman G M 1988 Biochemistry 273433

Fendler J H 1980 Acc. Chem. Res. 137

Fendler J H 1982 Membrane mimetic chemistry (New York: J Wiley)

Fendler J H 1984 Science 223888

Fuhrhop J H and Helfrich W 1993 Chem. Rev. 931565 
Fuhrhop J H and Mathieu J 1984 Angew Chem., Int. Ed. Engl. 23100

Fuhrhop J H, Schneider P, Boekma E and Helfrich W 1988 J. Am. Chem. Soc. 1102861

Fuhrhop J H, Suenson S, Boettcher C, Rossler E and Vieth H-M 1990 J. Am. Chem. Soc. 1124307

Fukuda H, Okuda H and Regen S L $1990 \mathrm{~J}$. Am. Chem. Soc. 1121635

Gaber B P 1990 Naval Research Reviews, Office of Naval Research, USA, vol. 42, one/1990

Ghyczy M and Niemann R 1992 In Skin and liposomes RPR Nattermann Phospholipid GmbH, Scientific

Publication No. 1

Gregoriadis G (ed.) 1988 Liposomes as drug carriers: Recent trends and progress (Chichester: Wiley \& Sons)

Gregoriadis G 1994 The biochemist Feb/Mar, p. 8

Hamachi I, Noda S and Kunitaka T 1990 J. Am. Chem. Soc. 1126744

Hamachi I, Noda S and Kunitake T 1991 J. Am. Chem. Soc. 1139625

Huang C and Mason J T 1986 Biochim. Biophys. Acta 864423

Ishikawa Y, Nishimi T and Kunitake T 1990 Chem. Lett. 25

Junginger H, Hofland H E and Bouwstra J A 1991 Cosmet. Toiletries 10645

Kaler E W, Murthy A K, Rodriguez B E and Zasadzinski J A N 1989 Science 2451371

Knox B E, Bhattacharya S, Ridge K D and Khorana $H$ G (in preparation)

Koynova R D, Kuttenreich H L, Tenchor B G and Hinz J -J 1988 Biochemistry 274612

Kunitake T 1992 Angew. Chem., Int. Ed. Engl. 31 709, and references therein

Kunitake T and Okahata Y 1977 J. Am. Chem. Soc. 993860

Kunitake Y, Okahata Y, Shimomura M, Yasunani S and Takarabe K $1981 \mathrm{~J}$. Am. Chem. Soc. 1035401

Kunitate T, Yamada N and Fukunaga N 1984 Chem. Lett. 1089

Lehn J-M 1988 Angew. Chem., Int. Ed. Engl. 2789

Lockhoff O 1991 Angew. Chem., Int. Ed. Engl. 30 1611, and reference therein

McElhaney R N 1986 Biochim. Biophys. Acta 864361

Menger F M, Chen X Y, Brocchini S, Hopkins H P and Hamilton D $1993 \mathrm{~J}$. Am. Chem. Soc. 1156600

Merkowitz M and Singh A 1991 Langmuir 716

Murakami Y and Kikuchi J 1991 In Bioorganic chemistry frontiers (ed.) H Dugas (New York: SpringerVerlag) vol. 2, p. 75

Murakami Y, Nakano A and Fukuya K $1980 \mathrm{~J}$. Am. Chem. Soc. 1024235

Nakashima N, Asakama S and Kunitake T $1989 \mathrm{~J}$. Am. Chem. Soc. 107509

Paulson H, Pluhaupt K W and Pigman W 1980 In The glyconjugates (ed.) D Horton (New York: Academic Press) vol. 1B

Paulson J C 1985 The receptors (ed.) M Coun (New York: Academic Press) vol. 2

Raison J K 1980 Membrane lipids: Structure and function. In The biochemistry of plants (ed.) P K Stumpf (New York: Academic Press) vol. 14, p. 57

Ringsdorf H, Schlarb B and Venzmer J 1988 Angew. Chem., Int. Ed. Engl. 27113

Rupert L A M, Engberts J B F N and Hoekstra D 1986 J. Am. Chem. Soc. 1083920

Schleicher A, Franke R, Hofmann K P, Finkelmann H and Welte W 1987 Biochemistry 265908

Schnur J M 1993 Science 2621669

Shobha J and Balasubramanian D 1986 J. Phys. Chem. 902800

Shobha J, Srinivas V and Balasubramanian D 1989 J. Phys. Chem. 9320

Silvius J R 1991 Chem. Phys. Lipids 57241

Singh A, Schoen A E and Schnur J M 1988 Chem. Commun. 1222

Slater J L and Huang C N 1992 In The structure of biological membranes (ed.) P Yeagle (Boca Raton, FL: CRC Press) pp. 175-210

Small D M 1986 Physical chemistry of lipids (New York: Plenum)

Smith J G, Walzen R L and German J B 1994 Biochem. Biphys. Acta 1154327

Sudholter E J R, Engberts J B F N and Hoekstra D 1980 J. Am. Chem. Soc. 1022467

Sundar C S, Raman B and Balasubramanian D 1991 Biochim. Biophys. Acta 106535

Surolia A and Bachhawat B K 1977 Biochim. Biophys. Acta 497760

Tyminski P N, Latimer L H and O'Brien D 1988 Biochemistry 272696

Uoeka R, Matsumoto Y, Harda K, Akahoshi H, Ihara Y and Kato Y 1992 J. Am. Chem. Soc. 1148339

Ueoka R, Matsumoto Y, Moss R A, Swarup S, Sugi A, Harda K, Kuchi J I and Murakami Y 1988 J. Am. Chem. Soc. 1101588

Yanagawa H, Ogawa Y, Furuta H and Tsuno K 1989 J. Am. Chem. Soc. 1114567

Zana R and Talmon Y 1993 Nature (London) 362 228, and references therein 